\title{
Técnicas nucleadoras na restauração de floresta ribeirinha em área de Floresta Ombrófila Mista, Sul do Brasil
}

\author{
Deisy Regina Tres* \\ Ademir Reis \\ Laboratório de Restauração Ambiental Sistêmica \\ Departamento de Botânica, Universidade Federal de Santa Catarina \\ Caixa Postal 476, CEP 88010-970, Florianópolis, SC - Brasil \\ *Autor para correspondência \\ tres_deisy@yahoo.com.br
}

Submetido em 04/02/2009

Aceito para publicação em 27/07/2009

\section{Resumo}

Em função de sua significativa importância no histórico de ocupação da região Sul do Brasil, a Floresta Ombrófila Mista, especialmente no Planalto Norte Catarinense, foi alvo de intenso processo de extrativismo, substituição da cobertura vegetal original por áreas agrícolas e pastagens. Atualmente sofre outro grande impacto que são os reflorestamentos homogêneos com espécies do gênero Pinus. O quadro atual caracteriza-se pela necessidade da restauração da conectividade local da paisagem, no sentido de restaurar as áreas ribeirinhas degradadas, buscando refazer níveis de conectividade entre os fragmentos e as áreas a restaurar. Este estudo investigou o papel do banco e da chuva de sementes de fragmentos ribeirinhos conservados adjacentes às áreas degradadas e a eficácia de técnicas nucleadoras na restauração das áreas ribeirinhas degradadas em fazendas produtoras de Pinus taeda L. Foram coletadas amostras do banco e da chuva de sementes de fragmentos conservados e implantadas técnicas de transposição de solo e poleiros artificiais nas áreas abertas degradadas. As áreas ribeirinhas mostraram potencial para dar início ao processo sucessional secundário, permitindo a formação de fases sucessionais iniciais. O uso de técnicas nucleadoras mostrou a possibilidade de acelerar o processo sucessional e indicaram a importância de estabelecer pontos de ligação entre áreas abertas e fragmentos conservados.

Unitermos: banco de sementes, chuva de sementes, nucleação, poleiros artificiais, transposição de solo

\section{Abstract}

Nucleation procedures in the restoration of riverine areas of the Mixed Rain Forest, Southern Brazil. Due to its significant importance in the history of the occupation of Southern Brazil, the mixed rain forest, particularly in the Planalto Norte Catarinense, was subjected to intense exploitation as well as the replacement of its original vegetation cover by pasture and agricultural areas. Nowadays, it suffers another great impact which is the homogeneous reforestation with species of Pinus. The present situation is characterized by the need for restoration of the local landscape's connectivity, which means restoring degraded riverine areas by repairing the connectivity between original fragments and areas to be restored. This study investigated the role of the seed bank and seed rain of preserved adjacent riverine fragments and the efficiency of nucleation procedures in the restoration of degraded riverine areas in Pinus taeda L. producing farms. Samples of the seed bank and seed rain 
of preserved fragments were collected and techniques of soil transposition and artificial perches were applied in the open degraded areas. The riverine areas demonstrated the potential to initiate the secondary succession process, allowing the formation of initial succession stages. The use of nucleation procedures showed the possibility of accelerating the succession process and indicated the importance of establishing linkage points between open areas and conserved remnants.

Key words: artificial perches, nucleation, seed bank, seed rain, soil transposition

\section{Introdução}

A Floresta Ombrófila Mista - FOM ou Floresta com Araucária é uma das mais exuberantes formações vegetacionais do Sul do Brasil. Ela abriga a principal conífera de interesse econômico de ocorrência subtropical no hemisfério Sul do continente americano, o pinheirobrasileiro ou pinheiro-do-Paraná (Araucaria angustifolia (Bert.) O. Kuntze.).

Em função da significativa importância no histórico de ocupação da região Sul do Brasil, não somente pela extensão territorial que ocupava, mas principalmente pelo valor econômico que representou durante quase um século (Klein, 1985), esta tipologia florestal foi alvo de intenso processo de extrativismo e substituição da cobertura vegetal original por atividades agrícolas, pecuárias e de silvicultura, além do crescimento das zonas urbanas (Fundação SOS Mata Atlântica/ Instituto Nacional de Pesquisas Espaciais/Instituto Socioambiental, 1998).

Considerando uma escala regional, o Planalto Norte Catarinense apresenta um histórico de uso e ocupação territorial baseado na redução em larga escala da Floresta Ombrófila Mista, especialmente a partir da década de setenta com o surgimento da atividade silvicultural através da introdução de espécies do gênero Pinus. Este cenário de substituição de florestas nativas por talhões plantados do pinheiro-americano representou um significativo aumento da fragmentação e degradação das áreas ribeirinhas e outras de preservação permanente, transformando a paisagem regional, maximizando as áreas produtivas em detrimento das áreas naturais $\mathrm{e}$ reduzindo e modificando a matriz florestal. O quadro atual caracteriza-se pela necessidade da restauração da conectividade local da paisagem (Tres e Reis, 2007), no sentido de restaurar as áreas ribeirinhas degradadas, buscando conectar fragmentos e as áreas a restaurar, direcionando a comunidade para a sua integração com a paisagem que a rodeia.

Em paisagens fragmentadas, as possibilidades de recolonização dependem da conectividade dos fragmentos, ou seja, da capacidade desses fragmentos receberem fluxos biológicos de fragmentos vizinhos (Cubina e Aide, 2001; Moilanen e Hanski, 2001). Fragmentos adjacentes às áreas degradadas representam a melhor fonte de propágulos para a regeneração natural e início do processo sucessional secundário, uma vez que são reconhecidos como núcleos de sucessão estocástica (Parker e Picket, 1999). Nestes núcleos ocorrem processos ecológicos chave para a manutenção da dinâmica das comunidades naturais. $\mathrm{O}$ aporte de sementes destes "núcleos históricos", através do banco e da chuva de sementes, potencializa a sucessão local através da dispersão alóctone e autóctone de sementes, consequentemente, aumentando a probabilidade de diversidade genética regional através do fluxo gênico (Tres et al., 2005; Reis e Tres, 2007).

Em função do mosaico produzido pela paisagem ser bastante heterogêneo, uma complexidade de condições naturais poderá ser potencializada, através da introdução de diversos elementos (solo, seres vivos...) desses núcleos históricos nas áreas a serem restauradas.

Dentro deste contexto, a aplicação da teoria da nucleação, proposta por Yarranton e Morrison (1972) surge como um princípio básico para atrair a diversidade e funcionalidade local para a área degradada. $\mathrm{O}$ processo de nucleação descrito pelos autores descreveu a dinâmica espacial da sucessão primária (formação de áreas naturais) em dunas canadenses. A vegetação de dunas iniciou com um estágio campestre com núcleos esparsos de uma espécie facilitadora, Juniperus spp. e espécies herbáceas agregadas; passou para um estágio intermediário com o desenvolvimento de núcleos de Quercus spp. que só mais tarde, culminaram na formação 
de florestas longevas de carvalho e seu sub-bosque associado, em áreas mais distantes do mar.

Desta forma, podemos hipotetizar que os fragmentos conservados adjacentes às áreas a serem restauradas funcionam como núcleos de diversidade biótica e abiótica, capazes de propiciar a formação de novas populações dentro de comunidades em restauração, facilitando a criação de novos nichos de regeneração/ colonização.

Nesse sentido, a nucleação representa uma alternativa diferenciada de restauração, uma vez que promove "gatilhos ecológicos" (Bechara, 2006) para a formação de comunidades estáveis e permite que os fenômenos eventuais (não normais) (Grant, 1980) através da sucessão natural possam atuar no sentido de integrar a área degradada com os fluxos naturais das áreas vizinhas.

Inspirados na teoria de nucleação, Reis et al. (2003) simularam a dinâmica espacial da natureza instituindo as técnicas nucleadoras. Estas representam um conjunto mínimo de interferências locais que objetivam uma sucessão de caráter natural, formando uma comunidade condizente com as novas condições das atuais paisagens fragmentadas. Dentro desta perspectiva, este estudo investigou o papel e a importância do banco e da chuva de sementes de fragmentos ribeirinhos conservados adjacentes às áreas degradadas e a eficácia de técnicas nucleadoras na restauração das áreas ribeirinhas degradadas nas fazendas produtoras de Pinus taeda L. Especificamente, buscamos responder algumas questões: Qual a capacidade de regeneração dos remanescentes ribeirinhos nas fazendas produtoras de Pinus? As técnicas de transposição de solo e de poleiros artificiais atraem diversidade para as áreas degradadas?

\section{Material e Métodos}

\section{Local de estudo}

O experimento foi conduzido na cidade de Rio Negrinho, Planalto Norte Catarinense, Sul do Brasil, em uma fazenda produtora de Pinus taeda L. de propriedade da Empresa Madeireira Battistella Florestal. Em julho de 2004 foi implantada uma Unidade Demonstrativa de Restauração Ambiental na fazenda Santa Alice (26²7'53"S, 49³1'31'W) ao longo da microbacia do Rio Verde. A microbacia tem suas nascentes na Fazenda Santa Alice e desemboca no Rio Corredeiras. Está inserida na Bacia Hidrográfica do Rio Iguaçu (Santa Catarina, 1986).

Originalmente a microbacia do Rio Verde era coberta pela Floresta Ombrófila Mista. Entretanto, ao longo dos anos, a fisionomia característica dessa formação foi modificada em virtude do processo de exploração dos recursos naturais, pelo extrativismo vegetal, posteriormente pelo desenvolvimento de atividades agropecuárias e, atualmente, pelo desenvolvimento da atividade silvícola (cultivo de Pinus). No início da década de sessenta, com a implementação de projetos de reflorestamento com o incentivo do Estado (Lei $\mathrm{n}^{\mathrm{o}} 5106 / 66$, que oferecia desconto no imposto de renda para iniciativas de reflorestamento), empresas madeireiras iniciaram o cultivo de Pinus taeda L. para o abastecimento de suas indústrias, realizando o reflorestamento em larga escala. A partir de 1965, do ponto de vista da Legislação Federal Ambiental, o Código Florestal, Lei $n^{\circ} 4.771$, estabeleceu uma faixa mínima a ser mantida para proteger a vegetação ao longo dos rios, considerando essas áreas de preservação permanente. No início, a lei estabelecia que para rios de até $10 \mathrm{~m}$ de largura, $5 \mathrm{~m}$ de faixa de vegetação deveriam ser preservados. Em 1986 e 1989 foram realizadas várias modificações na lei, ampliando a área de preservação permanente, esta condicionada à largura do rio. Referindo-se a rios de até $10 \mathrm{~m}$ de largura, ainda que os $5 \mathrm{~m}$ anteriormente protegidos por lei tivessem sido preservados, a partir da modificação da lei foram acrescentados $25 \mathrm{~m}$ de faixa de preservação permanente. Dessa forma, segundo a lei, $30 \mathrm{~m}$ ao longo dos cursos d'água devem ser preservados.

Dentro desse contexto se insere a microbacia do Rio Verde, onde foi preservada uma faixa contínua de $5 \mathrm{~m}$ de vegetação às margens do rio de mesmo nome, fruto da primeira redação do Código Florestal. Na faixa dos $25 \mathrm{~m}$ houve plantio de Pinus taeda L. em toda extensão da microbacia, acostado à legislação vigente da época. Pelas alterações realizadas no Código, no presente essa área é 
considerada de preservação permanente e a vegetação nativa deve ser restaurada. Em 2002 os talhões de Pinus taeda L. plantados na faixa dos $25 \mathrm{~m}$ foram retirados para permitir a restauração da vegetação ribeirinha, sendo, para tanto, criada a Unidade Demonstrativa (UD) de Restauração Ambiental na microbacia do Rio Verde, constituindo uma área piloto. A UD consiste de 19,54ha de áreas de preservação permanente (faixa de $5 \mathrm{~m}$ ), 9,94ha de áreas a restaurar (faixa de $25 \mathrm{~m}$ ) e uma área ribeirinha dentro de um fragmento conservado de 1,35ha. Na UD foram realizados estudos visando avaliar a capacidade de regeneração natural dos fragmentos ribeirinhos (banco e chuva de sementes) e a eficácia das técnicas nucleadoras para a restauração das florestas ribeirinhas (transposição de solo e poleiros artificiais).

\section{Avaliação da regeneração natural}

\section{a) Banco de sementes}

Foram coletadas quatro amostras de solo de $1 \mathrm{~m}^{2} \mathrm{e}$ profundidade de cerca $10 \mathrm{~cm}$ (incluindo a serapilheira), com espaçamento de $1 \mathrm{~m}$ entre elas em três pontos (unidade amostral) inseridos dentro da área dos $5 \mathrm{~m}$, cuja vegetação possui fitofisionomia predominantemente arbórea, com altura de 7 a $8 \mathrm{~m}$. As coletas foram realizadas em quatro períodos (meses): agosto e novembro de 2004 e fevereiro e abril de 2005. Ao final de um ano foram coletadas doze amostras de solo, sendo realizadas três réplicas por unidade amostral. As amostras recolhidas foram acondicionadas em sacos plásticos, peneiradas (malha de $5 \mathrm{~mm}$ ) e levadas à casa de vegetação. $\mathrm{O}$ método de avaliação adotado foi o de emergência de plântulas (Christoffoleti e Caetano, 1998). A riqueza de espécies e a densidade de sementes. $\mathrm{m}^{-2}$ total foram quantificadas. Foi levantado o percentual de espécies por forma de vida, estratégias de polinização e dispersão (Pijl, 1982). A fim de estimar a heterogeneidade do banco de sementes, foi realizada a comparação da composição de espécies por unidade amostral através do índice qualitativo de similaridade de Jaccard (Ludwig e Reynolds, 1988). Para comparar a composição de espécies e densidade de sementes. $\mathrm{m}^{-2}$ total por unidade amostral foram realizadas análises de variância e quando constatadas diferenças significativas entre grupos, o teste de separação de médias de Tukey ( $\alpha=0,05$ para todos testes) (Beiguelman, 1991).

\section{b) Chuva de sementes}

Foram fixados coletores permanentes de sementes (molduras de madeira de $1 \mathrm{~m}^{2}$ com fundo de sombrite, malha $5 \mathrm{~mm}$, a $1 \mathrm{~m}$ de altura do solo), espacialmente, nas seguintes áreas de coleta: 3 coletores na área ribeirinha conservada (faixa de $5 \mathrm{~m}$ ), 3 coletores na área ribeirinha a restaurar (aberta de $25 \mathrm{~m}$ ) e 3 coletores na área ribeirinha dentro de um fragmento conservado adjacente às áreas a restaurar. Os coletores foram instalados em julho de 2004 e o material captado foi recolhido mensalmente, pelo período de um ano (agosto de 2004 a julho de 2005), totalizando 12 réplicas temporais. As sementes capturadas foram depositadas em bandejas com areia esterilizada e levadas à casa de vegetação. $\mathrm{O}$ método de avaliação adotado foi o de emergência de plântulas (Christoffoleti e Caetano, 1998). A composição de espécies e a densidade de sementes. $\mathrm{m}^{-2}$ total foram quantificadas. Foi levantado o percentual de espécies por forma de vida, estratégias de polinização e dispersão. Registrou-se a composição de espécies e densidade de sementes. $\mathrm{m}^{-2}$ total por mês de coleta. A fim de estimar a heterogeneidade da chuva de sementes, foi realizada a comparação da composição de espécies por área de coleta através do índice qualitativo de similaridade de Jaccard (Ludwig e Reynolds, 1988). Para comparação das diferentes áreas de coleta em relação à composição de espécies e a densidade de sementes. ${ }^{-2}$ classificadas por forma de vida, estratégias de polinização e dispersão foi aplicado o teste $\chi^{2}$ de contingência, ao nível de significância de 5\% (Sokal e Rohlf, 1995).

\section{Avaliação das técnicas nucleadoras}

\section{a) Transposição de solo}

Foram retiradas amostras de solo de $1 \mathrm{~m}^{2} \mathrm{e}$ profundidade de cerca $10 \mathrm{~cm}$ (incluindo a serapilheira) em três pontos (unidades amostrais) localizados dentro das áreas ribeirinhas de $5 \mathrm{~m}$. Em cada unidade amostral foram coletadas quatro amostras de solo, com espaçamento de $1 \mathrm{~m}$ entre elas. As amostras recolhidas foram acondicionadas em sacos plásticos e levadas à área dos $25 \mathrm{~m}$. Nesta área foram delimitadas unidades de $1 \mathrm{~m}^{2}$, com espaçamento de $1 \mathrm{~m}$ entre cada unidade amostral. Para transposição, a camada de serapilheira 
foi retirada até aparecer o solo, sobre o qual foram depositadas as amostras coletadas anteriormente. Estas foram espalhadas uniformemente sobre o solo dentro da medida estabelecida, de maneira a formar núcleos. As transposições foram realizadas em quatro períodos (meses): agosto e novembro de 2004 e fevereiro e abril de 2005. Ao final foram transpostas doze amostras de solo de $1 \mathrm{~m}^{2}$, sendo realizadas três réplicas por período. As avaliações foram realizadas a cada três meses, identificando-se as plântulas recrutadas na área transposta. É importante salientar que neste procedimento não houve diferenciação da origem das plântulas recrutadas, se autóctones (da própria área) ou alóctones (do solo transposto). Não foram utilizadas amostras controle ou testemunha. Após um ano de avaliação foram listadas as espécies recrutadas a partir desta técnica. Foi levantado o percentual de espécies por forma de vida, estratégia de polinização e dispersão (Pij1, 1982).

\section{b) Poleiros artificiais}

Dentro da área ribeirinha de $25 \mathrm{~m}$, foram instalados seis poleiros do tipo "seco" com coletores de sementes fixados em suas bases, sendo dois poleiros em cada uma das três áreas; e seis coletores que serviram como testemunha, dispostos a uma distância de 50m dos poleiros secos. Os poleiros secos são estruturas que imitam galhos secos e que tem a função de servir como ponto de pouso para a avifauna. Estes foram montados com três varas de bambu, enterradas e amarradas entre si, sendo deixadas as ramificações laterais superiores. Os coletores permanentes de sementes foram confeccionados conforme o item "Chuva de sementes". Os poleiros e coletores foram instalados em julho de 2004 e o material captado foi recolhido, mensalmente, pelo período de um ano (setembro de 2004 a agosto de 2005). As sementes coletadas foram levadas à casa de vegetação para avaliação através da emergência de plântulas. A composição de espécies e a densidade de sementes. $\mathrm{m}^{-2}$ total foram quantificadas. Foi levantado o percentual de espécies por forma de vida, estratégias de polinização e dispersão. A comparação da riqueza (número) de espécies foi realizada através do índice qualitativo de similaridade de Jaccard (Ludwig e Reynolds, 1988). Para comparação dos poleiros artificiais e controles, em relação à composição total de espécies, densidade total de sementes. $\mathrm{m}^{-2}$, bem como a composição de espécies classificadas por forma de vida, estratégias de polinização e dispersão foi aplicado o teste- $t$, a um nível de significância de 5\% (Ferreira, 1991).

\section{Resultados}

\section{Avaliação da regeneração natural}

\section{a) Banco de sementes}

$\mathrm{Na}$ avaliação do banco de sementes foram identificadas 115 espécies, num total de 27.273 plântulas em $12 \mathrm{~m} 2$ de solo. O banco de sementes apresentou espécies com todas as formas de vida, com predomínio de ervas (47\%), seguido de arbustos (21\%), árvores (9\%), lianas (7\%) e indeterminadas (16\%). Foram registradas, quanto à estratégia de dispersão, $60 \%$ de espécies anemocóricas, 19\% zoocóricas e 5\% autocóricas; e quanto à estratégia de polinização, $68 \%$ de espécies zoofílicas e 16\% de espécies anemofílicas.

As Tabelas 1 e 2 apresentam o índice de similaridade entre os três pontos de coleta do banco de sementes e a densidade de sementes $\mathrm{m}^{-2}$ e composição de espécies em cada ponto de coleta, respectivamente.

TABELA 1: Similaridade entre composição de espécies do banco de sementes obtida em três amostras dentro da área ribeirinha de $5 \mathrm{~m}$ na Unidade Demonstrativa de Restauração Ambiental da Microbacia do Rio Verde, Rio Negrinho, $\mathrm{SC}$; onde $\mathrm{PC}=$ ponto de coleta.

\begin{tabular}{ccc}
\hline $\begin{array}{c}\text { Pontos de Coleta e Exclu- } \\
\text { sividade de Espécies }\end{array}$ & $\begin{array}{c}\text { Espécies em } \\
\text { Comum }\end{array}$ & Jaccard \\
\hline PC 1 (39) x PC 2 (14) & 37 & 0,41 \\
PC 1 (29) x PC 3 (34) & 47 & 0,42 \\
PC 2 (16) x PC 3 (45) & 35 & 0,36 \\
\hline
\end{tabular}


TABELA 2: Densidade de sementes. $\mathrm{m}^{-2}$ e composição de espécies detectadas em amostras de solo coletado dentro da área ribeirinha de $5 \mathrm{~m}$ na Unidade Demonstrativa de Restauração Ambiental da Microbacia do Rio Verde, Rio Negrinho, SC; onde PC = ponto de coleta.

\begin{tabular}{ccc}
$\begin{array}{c}\text { Pontos de } \\
\text { Coleta }\end{array}$ & $\begin{array}{c}\text { Composição de } \\
\text { espécies* }\end{array}$ & $\begin{array}{c}\text { Densidade de } \\
\text { sementes.m }^{-2 *}\end{array}$ \\
\hline PC 1 & $35,5 \pm 7,4 \mathrm{a}$ & $1141 \pm 577,9 \mathrm{ab}$ \\
PC 2 & $24,3 \pm 9,7 \mathrm{a}$ & $773,5 \pm 592,4 \mathrm{~b}$ \\
PC 3 & $39,5 \pm 13,4 \mathrm{a}$ & $4903,7 \pm 3334,9 \mathrm{a}$ \\
\hline
\end{tabular}

*Médias e desvio padrão de quatro repetições seguidas pela mesma letra na coluna não diferem entre si de acordo com o teste de Tukey $(P \leq 0,05)$.

\section{b) Chuva de sementes}

$\mathrm{Na}$ avaliação da chuva de sementes identificou-se 61 espécies, num total de 747 plântulas em $9 \mathrm{~m}^{2}$. A chuva de sementes apresentou espécies com todas as formas de vida, com predomínio de ervas (42\%), seguido de árvores $(20 \%)$, arbustos $(15 \%)$, lianas $(5 \%)$ e indeterminadas $(18 \%)$. Foram registradas quanto à estratégia de dispersão, 57\% de espécies anemocóricas, 18\% zoocóricas, 7\% autocóricas; e quanto à estratégia de polinização, $62 \%$ de espécies zoofílicas e $20 \%$ anemofílicas.

Registrou-se a ocorrência de sementes durante todos os meses de coleta, indicando que a chuva de sementes é contínua na área (Figura 1). Houve uma correlação positiva entre a composição de espécies e densidade de sementes. $\mathrm{m}^{-2}$. Ocorreu porém, uma variação temporal tanto na densidade de sementes quanto na composição de espécies com dois picos de deposição de sementes, um que se inicia no final da primavera e máximos no verão e outro no inverno. Entretanto, a deposição de sementes nos meses mais quentes foi superior à observada no inverno. A riqueza de espécies variou entre 23 espécies (mês de janeiro) e 2 espécies (mês de maio).

A Tabela 3 apresenta o índice de similaridade entre os três pontos de coleta da chuva de sementes.

TABELA 3: Similaridade entre composição florística entre diferentes áreas de coleta da chuva de sementes na Unidade Demonstrativa de Restauração Ambiental da Microbacia do Rio Verde, Rio Negrinho, SC; onde A1=área ribeirinha aberta de $25 \mathrm{~m}, \mathrm{~A} 2=$ área ribeirinha conservada de $5 \mathrm{~m}, \mathrm{~A} 3=$ área ribeirinha dentro de fragmento conservado.

\begin{tabular}{ccc}
\hline $\begin{array}{c}\text { Áreas de Coleta e Exclusi- } \\
\text { vidade de Espécies }\end{array}$ & $\begin{array}{c}\text { Espécies em } \\
\text { Comum }\end{array}$ & Jaccard \\
\hline A1 (14) x A2 (24) & 15 & 0,28 \\
A1 (15) x A3 (16) & 14 & 0,31 \\
A2 (20) x A3 (11) & 19 & 0,38 \\
\hline
\end{tabular}

Espacialmente, a densidade de sementes. $\mathrm{m}^{-2}$ e a riqueza de espécies foram variáveis. Detectaram-se 11 espécies comuns às três áreas de coleta. As áreas de coleta 1, 2 e 3 apresentaram 30\% (29 espécies); 40\% (39 espécies); 31\% (30 espécies) da ocorrência total de espécies, respectivamente. Quanto à densidade de sementes. $\mathrm{m}^{-2}$, a área ribeirinha dentro do fragmento

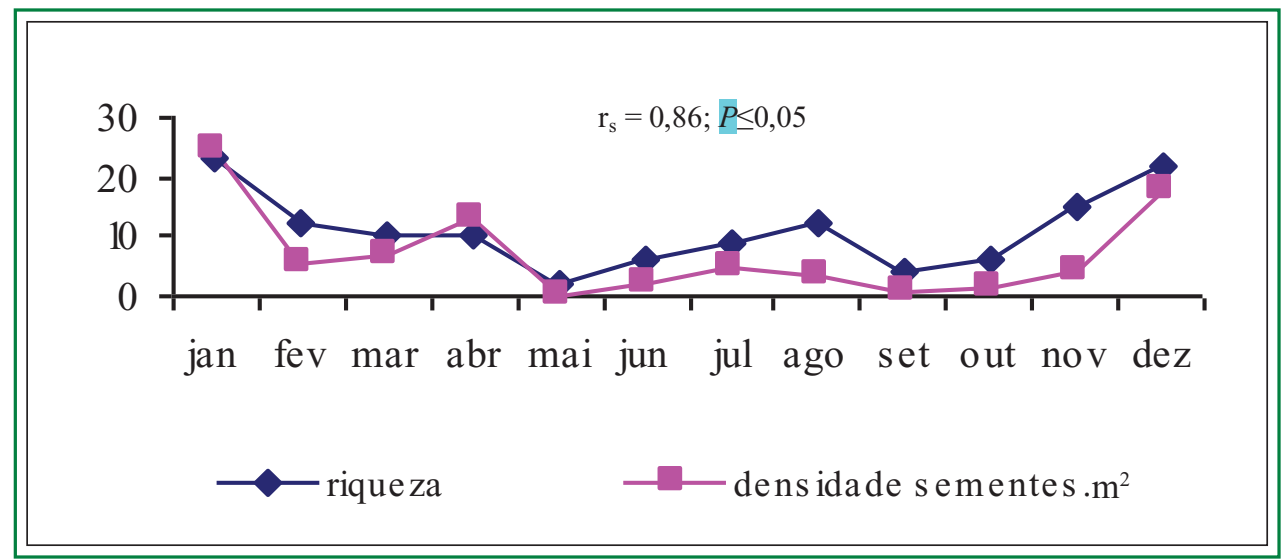

FIGURA 1: Riqueza de espécies e densidade de sementes. $\mathrm{m}^{-2}$ detectadas na chuva de sementes captada na Unidade Demonstrativa de Restauração Ambiental da Microbacia do Rio Verde, Rio Negrinho, SC. 
conservado foi a que prevaleceu sobre as outras, apresentando $43 \%$, seguida da área conservada conservada de $5 \mathrm{~m}$ que apresentou $32 \%$, enquanto que a área ribeirinha aberta de $25 \mathrm{~m}$ apresentou $24 \%$ da densidade total de sementes registrada.

A Tabela 4 apresenta os resultados obtidos a partir das comparações entre as áreas de coleta, quanto à composição de espécies e densidade de sementes.m ${ }^{-2}$, por hábito, estratégias de dispersão e polinização.

TABELA 4: Riqueza de espécies (S) e densidade de sementes. $\mathrm{m}^{-2}$ (D) por hábito, estratégias de dispersão e polinização, registradas sob as condições: área ribeirinha aberta de $25 \mathrm{~m}$ (A1), área ribeirinha conservada de $5 \mathrm{~m}$ (A2), área ribeirinha dentro de fragmento conservado (A3), na Unidade Demonstrativa de Restauração Ambiental da Microbacia do Rio Verde, Rio Negrinho, SC.

\begin{tabular}{|c|c|c|c|c|c|c|}
\hline \multicolumn{7}{|c|}{ Áreas de Coleta } \\
\hline \multirow{2}{*}{$\begin{array}{c}\text { Características } \\
\text { Ecológicas }\end{array}$} & \multicolumn{2}{|c|}{ A1xA2 } & \multicolumn{2}{|c|}{$\mathbf{A 1 x A 3}$} & \multicolumn{2}{|c|}{ A2xA3 } \\
\hline & S & $\mathrm{D}$ & S & $\mathrm{D}$ & $\mathrm{S}$ & $\mathrm{D}$ \\
\hline Erva & $\approx$ & $\mathrm{A} 2>\mathrm{A} 1$ & $\approx$ & $\approx$ & $\approx$ & $\mathrm{A} 2>\mathrm{A} 3$ \\
\hline Liana & $\approx$ & $\approx$ & $\approx$ & $\approx$ & $\approx$ & $\approx$ \\
\hline Arbusto & $\approx$ & $\mathrm{A} 1>\mathrm{A} 2$ & $\approx$ & $\mathrm{A} 1>\mathrm{A} 3$ & $\approx$ & $\mathrm{A} 3>\mathrm{A} 2$ \\
\hline Árvore & $\approx$ & $\mathrm{A} 2>\mathrm{A} 1$ & $\approx$ & $\mathrm{A} 3>\mathrm{A} 1$ & $\approx$ & $\mathrm{A} 3>\mathrm{A} 2$ \\
\hline Anemocoria & $\approx$ & $\approx$ & $\approx$ & $\approx$ & $\approx$ & $\approx$ \\
\hline Autocoria & $\approx$ & $\approx$ & $\approx$ & $\approx$ & $\approx$ & $\approx$ \\
\hline Zoocoria & $\approx$ & $\mathrm{A} 2>\mathrm{A} 1$ & $\approx$ & $\mathrm{A} 3>\mathrm{A} 1$ & $\approx$ & $\mathrm{A} 3>\mathrm{A} 2$ \\
\hline Anemofilia & $\approx$ & $\mathrm{A} 1>\mathrm{A} 2$ & $\approx$ & $\approx$ & $\approx$ & $\mathrm{A} 3>\mathrm{A} 2$ \\
\hline Zoofilia & $\mathrm{A} 2>\mathrm{A} 1$ & $\mathrm{~A} 2>\mathrm{A} 1$ & $\approx$ & $\mathrm{A} 3>\mathrm{A} 1$ & $\approx$ & $\approx$ \\
\hline
\end{tabular}

$\approx$ : não diferiram significativamente de acordo com o teste $\chi^{2}$; $P \leq 0,05$.

\section{Avaliação das técnicas nucleadoras}

\section{a) Transposição de solo}

Foram recrutadas 36 espécies em $12 \mathrm{~m}^{2}$ de solo em um período de um ano de avaliação. A transposição de solo apresentou espécies com todas as formas de vida, com predomínio de ervas (45\%), seguido de arbustos (32\%), árvores (16\%) e lianas (11\%). Foram registradas, quanto à estratégia de dispersão, $72 \%$ de espécies anemocóricas, 14\% zoocóricas e 14\% autocóricas; e quanto à estratégia de polinização, $86 \%$ de espécies zoofílicas e $14 \%$ de espécies anemofílicas.

\section{b) Poleiros artificiais}

Foram detectadas nos coletores de sementes sob poleiros artificiais 50 espécies, com $18 \%$ de espécies zoocóricas, confirmando o efeito dessas estruturas para atração da fauna dispersora. Destas, cinco são árvores, três arbustos e uma erva. Quanto às demais estratégias de dispersão, registraram-se $64 \%$ de espécies anemocóricas, $2 \%$ epizoocóricas e $2 \%$ autocóricas. Quanto à estratégia de polinização, 66\% das espécies detectadas são zoofílicas e $20 \%$ anemofílicas. A densidade total foi de 22,8 sementes. $\mathrm{m}^{-2}$. Comparando com a condição controle, a chuva de sementes sob poleiros artificiais apresentou diferença significativa em relação ao hábito árvore $(\mathrm{N}=6,6 ; t=2,35 ;$ g.l. $=1 ; P \leq 0,05)$, à estratégia de dispersão zoocórica $(\mathrm{N}=6,6 ; t=2,60 ;$ g.1. $=1 ; P \leq 0,05) \mathrm{e}$ à estratégia de polinização zoofílica $(\mathrm{N}=6,6 ; t=2,77$; g.1.=1; $P \leq 0,05)$ (Tabela 5).

Os poleiros artificiais apresentaram diferença significativa na composição total de espécies em relação às condições controle $(\mathrm{N}=6,6 ; t=2,48$; g.l. $=1$; $P \leq 0,05)$.

TABELA 5: Composição de espécies por hábito, estratégias de dispersão e polinização, captadas nas condições: poleiros artificiais (PA) e coletores controle (CC), amostradas na Unidade Demonstrativa de Restauração Ambiental da Microbacia do Rio Verde, Rio Negrinho, SC.

\begin{tabular}{|c|c|c|c|c|c|c|c|c|c|}
\hline \multicolumn{10}{|c|}{ Características ecológicas } \\
\hline & \multicolumn{4}{|c|}{ hábito* } & \multicolumn{3}{|c|}{ estratégias de dispersão* } & \multicolumn{2}{|c|}{ estratégias de polinização* } \\
\hline & Erva & Liana & Arbusto & Árvore & Anemocoria & Zoocoria & Autocoria & Anemofilia & Zoofilia \\
\hline PA & $10,0 \pm 3,0 \mathrm{a}$ & $0,1 \pm 0,4 \mathrm{a}$ & $3,0 \pm 1,7 \mathrm{a}$ & $3,1 \pm 1,3 \mathrm{a}$ & $12,5 \pm 4,3 \mathrm{a}$ & $3,1 \pm 1,2 \mathrm{a}$ & $0,5 \pm 0,6 \mathrm{a}$ & $4,8 \pm 1,0 \mathrm{a}$ & $11,3 \pm 4,5 \mathrm{a}$ \\
\hline $\mathbf{C C}$ & $7,1 \pm 2,4 \mathrm{a}$ & $0,1 \pm 0,4 \mathrm{a}$ & $1,8 \pm 1,2 \mathrm{a}$ & $1,6 \pm 0,8 b$ & $8,6 \pm 2,6 \mathrm{a}$ & $1,5 \pm 1,1 b$ & $0,6 \pm 0,5 \mathrm{a}$ & $4,6 \pm 1,2 \mathrm{a}$ & $6,1 \pm 0,8 b$ \\
\hline
\end{tabular}

* Médias e desvio padrão de seis repetições seguidas pela mesma letra na coluna não diferem entre si de acordo com o teste $t$ de Student $(P \leq 0,05)$. 


\section{Discussão}

De acordo com a SER - Society Ecological Restoration (2004), restauração ecológica é "the process of assisting the recovery of an ecosystem that has been degraded, damaged, or destroyed". Nesse sentido, a restauração ecológica é um processo que objetiva o desenvolvimento de um ecossistema resiliente e autosustentável com respeito a estrutura, composição de espécies e função (SER e IUCN, 2004).

Dentro destes conceitos, as áreas ribeirinhas da microbacia do Rio Verde possuem potencial para dar início ao processo sucessional secundário, permitindo a formação de fases sucessionais iniciais, cuja característica pioneira deve permitir a formação de séries sucessionais mais avançadas na área degradada. Espécies pioneiras, especialmente herbáceas e arbustivas, e de etapas intermediárias, quando recrutadas, devem ser capazes de modificar o ambiente, tanto biótico como abiótico, permitindo uma nova dinâmica sucessional. Desta forma, a fase inicial herbáceo-arbustivo predominante no banco e na chuva de sementes não deve ser menosprezada no processo de restauração ecológica da mata ribeirinha na microbacia do Rio Verde, pois é chave para por em marcha o processo sucessional e favorecer sua progressão até comunidades mais maduras

Diversos autores defendem que a maneira mais apropriada para restaurar é induzir um processo de sucessão secundária tão similar quanto possível aos processos naturais, formando comunidades que tendem a constituir estágios estáveis ao longo do tempo e do espaço (McIntosh, 1980; Bradshaw, 1983; Dobson et al., 1997; Palmer et al., 1997; Whisenant, 1999; Young, 2000; Young et al., 2001; Reis et al., 2003; Walker \& del Moral, 2003).

O predomínio de espécies zoofílicas, tanto no banco de sementes como na chuva de sementes, indica a importância desses elementos para dar início ao processo sucessional. Essas espécies têm potencial para atrair polinizadores para a área degradada, característica essencial numa fase inicial da sucessão secundária.

A variação sazonal na produção de sementes registrada a partir da coleta da chuva de sementes deste estudo deve influenciar a regeneração das espécies vegetais, representando uma importante estratégia no potencial da área, ao mesmo tempo em que representa uma distribuição contínua de recursos de frutos e sementes durante todo o ano para fauna. Coletar a chuva de sementes de fragmentos próximos, com periodicidade mensal, durante no mínimo um ano, é uma forma de buscar a diversidade na fenologia das espécies da região. Reis et al. (1999) sugerem a colocação de coletores de sementes permanentes dentro de comunidades vegetais conservadas como forma de coletar sementes durante todo $\mathrm{o}$ ano e de forma diversificada. Os autores sugerem que o material captado poderá ir direto para o campo, formando pequenos núcleos com folhas e sementes dentro das áreas degradadas.

A similaridade entre pontos de coleta do banco e da chuva de sementes caracteriza a heterogeneidade espacial e temporal da paisagem. Essa característica heterogênea deverá aumentar as probabilidades de recrutamento de diferentes espécies adaptadas a diversas condições bióticas e abióticas. Os mecanismos de regeneração estão contribuindo com importantes elementos heterogêneos para as diferentes etapas da sucessão natural, uma vez que representam distintas fontes de heterogeneidade, interagindo no sentido de produzirem um processo dinâmico de formação do meio ambiente, conforme defendido por Stewart et al. (2002). Essa heterogeneidade ambiental da paisagem deve aumentar as probabilidades de formação de diferentes habitats, estando significativamente correlacionada com a biodiversidade ambiental, como discutido por Rosenzweig (1995) e Wilson (2002).

$\mathrm{O}$ aporte de sementes nas áreas ribeirinhas em estudo está representando a potencialidade de sucessão local através da dispersão alóctone e autóctone de sementes e, consequentemente, o aumento da diversidade genética regional através do fluxo gênico. Esse fluxo de sementes tem capacidade de manter o dinamismo do banco de sementes e do banco de plântulas, dando continuidade ao processo sucessional.

O predomínio de espécies de fase inicial, tanto no banco como na chuva de sementes pode parecer uma opção pobre se a concepção de restauração estiver condicionada a uma visão de implantação de espécies arbóreas. No entanto, pensando na restauração como 
um processo de longo prazo, onde cada fase tem seu papel para a construção de comunidades, acelerar estágios representa perder funções irreversíveis durante o processo de restauração. Pular fases na construção de comunidades implica em dar direcionamentos diversos daqueles que correspondem ao potencial natural da paisagem. Zamora et al. (2004) investigando o potencial dos "matorrales" em florestas do Mediterrâneo, mostram que plantas pioneiras e de etapas intermediárias da sucessão são capazes de melhorar o êxito da restauração e favorecer a progressão até florestas maduras, uma vez que servem de facilitadoras para a regeneração de muitas espécies arbóreas.

No sentido de priorizar o restabelecimento das funções sobre a produtividade de biomassa, a manifestação das fases iniciais é a garantia de uma construção conjunta e equilibrada entre produtores, consumidores e decompositores, restaurando todos os componentes da comunidade, principalmente no que tange a riqueza de espécies e mudanças físicas e biológicas do solo.

Estes conceitos ilustram uma nova tendência da prática da restauração em priorizar modelos de conservação da biofuncionalidade por redirecionar a comunidade degradada em direção a integração com a paisagem que a rodeia, refletindo seus processos estocásticos e sua atual capacidade de resiliência (Whisenant, 1999; Garcia et al., 2000; Garcia e Zamora, 2003; Reis et al., 2003; Castro et al., 2004; GómezAparicio et al., 2004; Zamora et al. 2004; Griffith e Toy, 2005; Metzger, 2006; Bechara et al., 2007a; 2007b; Reis et al., 2007; Tres et al., 2007a; 2007b; Benayas et al., 2008).

A incorporação de conceitos de sucessão ecológica em trabalhos sobre restauração de áreas degradadas tem trazido significativas mudanças metodológicas para estas atividades. Uma delas é a sinalização da importância de se realizar diagnósticos das áreas em estudo, antes da aplicação de qualquer técnica de restauração.

O diagnóstico realizado neste estudo mostrou ser essencial para compreender alguns processos ecológicos nas áreas ribeirinhas, assim como estabelecer estratégias adequadas para a restauração daquele ambiente, uma vez que constitui parte do planejamento ambiental da paisagem. Avaliações do banco e chuva de sementes e do monitoramento da regeneração natural em diferentes ambientes brasileiros puderam inferir o potencial de resiliência das comunidades naturais em restinga (Bechara, 2003; Hmeljevski, 2004; Vieira, 2004; Espíndola, 2005), em Floresta Ombrófila Mista (Tres, 2006; Guinle, 2006), em Floresta Estacional Semidecidual e Cerrado (Bechara, 2006).

A proposta das técnicas nucleadoras é a utilização de mecanismos ecológicos que visem formar microhabitats em núcleos propícios para a ocorrência de uma série de condições oportunas para a regeneração natural, como a chegada de espécies vegetais de todas as formas de vida e formação de uma rede de interações entre os organismos, como defendida por Hurlbert (1971), Williams e Martinez (2000) e Bascompte et al. (2006).

Nesse sentido, as duas técnicas nucleadoras aplicadas neste estudo mostraram potencial para desencadear e conduzir um processo de sucessão nas áreas ribeirinhas de forma a atingir uma condição mais próxima possível das comunidades naturais.

A transposição de núcleos de solo trouxe para a área em estudo um novo banco de sementes que, devido ao seu revolvimento e transporte, favoreceu um rápido recrutamento de 36 espécies novas para o novo sítio. Estes núcleos tendem a se comportar como se fossem pequenos pedaços do ecossistema de origem, onde o banco de sementes aguarda oportunidade para que suas espécies sejam recrutadas. A função básica desta técnica é a introdução de espécies pioneiras que se desenvolvem e proliferam-se em núcleos, atraindo a fauna consumidora (herbívoros, polinizadores e dispersores de sementes), bem como preparando o ambiente para as seres subsequentes já que estas espécies entram em senescência precocemente e cumprem seu papel de facilitadoras. Neste estudo, espécies arbustivas pioneiras devem contribuir para a formação de um microclima adequado para que outras espécies possam se estabelecer, principalmente permitindo que a maciça cobertura de gramíneas, formada a partir da germinação de sementes que está presente no solo transposto, possa ser substituída por outras espécies, e integrada na biomassa do solo. 
Os núcleos formados devem gerar aglomerados de vegetação densa que se destacam na paisagem como os primeiros núcleos de abrigo para a fauna e produção das primeiras sementes na área em questão. Certamente, em curto prazo, os animais estarão fazendo a interligação entre os fragmentos conservados e as áreas em restauração, através do fluxo gênico (pólen e sementes), garantindo uma maior permeabilidade da paisagem no espaço e no tempo. Nesse sentido, as espécies arbustivas recrutadas a partir desta técnica devem ser visitadas por muitos pássaros onívoros, favorecendo a chegada de uma diversidade de espécies arbóreas para as áreas ribeirinhas em processo de restauração, advinda de fragmentos adjacentes (Reis et al., 1999; Tres et al., 2007). A manutenção desse estádio sucessional somado a das espécies herbáceas, representa uma grande probabilidade de garantir a resiliência na área em restauração.

Outra grande vantagem desta técnica consiste na heterogeneidade do material genético introduzido, desde que haja a preocupação de representar, nas amostras de solos, todos os fragmentos vizinhos da área a ser restaurada. Nesse sentido, as áreas restauradas deverão ter condições de modificar a paisagem regional, uma vez que as populações formadas tenderão a trocar material genético com as áreas vizinhas. Para isto, torna-se pertinente que, no diagnóstico a ser realizado antes da aplicação das ações restauradoras, sejam levantados todos os fragmentos da paisagem que se pretende conectar com a área a ser restaurada.

A formação de núcleos de solo facilitadores da sucessão foi testada com sucesso para o ambiente de restinga por Vieira (2004) com a introdução de 54 espécies em $16 \mathrm{~m}^{2}$ de solo e por Bechara (2006) em Floresta Estacional Semidecidual e Cerrado, com a introdução de 25 espécies em $12 \mathrm{~m}^{2}$ e 14 espécies em $20 \mathrm{~m}^{2}$ de solo, respectivamente. Basso et al. (2007) selecionaram um fragmento com um bracatingal com idade estimada de 10 anos, onde retiraram 50 núcleos de solo de bracatinga (Mimosa scabrella) de $1 \mathrm{~m}^{2} \mathrm{e}$ introduziram em áreas riberinhas degradadas em Floresta Ombrófila Mista. Aos seis meses de implantação dos núcleos de solo, 43 (88\%) apresentavam plântulas de bracatinga, com média de $125( \pm 45)$ indivíduos/ núcleo.
Um aspecto funcional importante da transposição de solo está na probabilidade de introdução na área em restauração de uma nova comunidade de microorganismos inseridos em núcleos de solo que irão dar início a uma nova sucessão edáfica. Provavelmente, as transposições de solo realizadas também adicionaram às áreas ribeirinhas uma diversidade de micro e meso organismos. Quando transpostos para a área degradada, estes organismos terão potencialidade para suprir elementos deficientes como carbono e nitrogênio, absorver nutrientes minerais, degradar matéria orgânica, fragmentar a serapilheira, atuar como patógenos vegetais, exercendo diversas atividades importantes para o equilíbrio biológico do solo (Moreira e Siqueira 2002).

Os poleiros secos, idealizados por Reis et al. (2003) são estruturas que imitam galhos secos de plantas e atuam como estrutura de repouso, forrageamento e caça para aves. Esta técnica vem ganhando espaço nas restaurações e resultados significativos foram obtidos por Espíndola (2005); Bechara (2006) e Tres (2006), que mostraram seu forte poder nucleador. Tres e Reis (2007) discutem o papel dos poleiros artificiais como estratégia para a conectividade da paisagem, atuando como trampolins ecológicos, especialmente quando dispersos numa matriz de não-habitat, favorecem os fluxos biológicos e potencializam a permeabilidade da matriz. Nesse estudo, os poleiros artificiais instalados nas áreas ribeirinhas mostraram o potencial de formação de novos núcleos de sementes. As concentrações de sementes servem, além de fonte de propágulos para a comunidade em processo de restauração, como núcleos de alimento para dispersores secundários e outros consumidores, como descrito pela teoria de saciação do predador de Janzen (1970). Esse processo possibilita a formação de uma nova cadeia trófica e aumenta a diversidade funcional da área, promovendo a reconstrução da comunidade em todos os seus elementos (produtores, consumidores e decompositores). É necessário frisar que, devido à alta concentração de sementes sob estes poleiros, estes são locais onde raramente ocorrerá recrutamento de plântulas, uma vez que representam locais de alta predação e de dispersão secundária das sementes aí depositadas. 
As espécies zoocóricas detectadas sob poleiros artificiais estão predominantemente associadas à forma de vida arbórea. A avifauna que visita essas estruturas parece ter hábito alimentar arborícola, uma vez que frequenta outros fragmentos e traz consigo sementes desses locais, contribuindo para aumentar a diversidade de formas de vida para a área degradada. É nesse sentido que os poleiros artificiais atuam como trampolins ecológicos entre os ambientes ribeirinhos isolados pela fragmentação, promovendo a conectividade da diversidade de mosaicos da paisagem.

$\mathrm{O}$ aspecto mais relevante dos poleiros artificiais se deve ao fato da chuva de sementes provocada por estas estruturas ser essencialmente de origem alóctone. Por outro lado, dependendo da distância entre os poleiros e as áreas fonte, os propágulos podem ser autóctones. Nesse sentido, a chegada de sementes de espécies diferentes ou mesmo das espécies locais, garante um novo fluxo gênico na área em restauração.

Espíndola et al. (2003) discutiram as formas e funções de poleiros artificiais, argumentando que esta técnica pode atrair uma variação maior de espécies, desde que sejam diversificados, de maneira a favorecer comportamentos animais distintos. Um exemplo é a implantação de poleiros vivos que imitam árvores vivas, os quais têm a função de atrair animais com comportamento distinto e que não utilizam os poleiros secos. Dentro desse grupo, destacam-se os morcegos, que procuram locais de abrigo para completarem a alimentação dos frutos colhidos em árvores distantes. Aves frugívoras também são atraídas por poleiros vivos quando estes fornecem fonte de alimento. A grande variação entre poleiros secos e vivos (estes também chamados de torres de cipó) implementa uma chuva de sementes bem mais diversificada e cumpre com maior eficiência o papel de trampolins ecológicos entre fragmentos. Os experimentos têm mostrado que atuam no sentido de atrair a fauna da vizinhança, quando estão distribuídos esparsamente.

No caso específico da restauração de áreas ribeirinhas em meio a plantações de Pinus recomendase a formação de poleiros através do anelamento de algumas árvores antes da retirada dos talhões. Estas plantas podem atuar como poleiros por um período bem maior de cerca de 5 anos (Reis e Tres, 2007).
Observações realizadas em formação de Cerrado e Floresta Estacional Semidecidual, mostraram que 27 e 35 espécies de aves utilizaram, respectivamente, os poleiros, sendo que mais de $50 \%$ destas eram dispersoras de sementes (Bechara, 2006).

A avaliação do banco e da chuva de sementes dos remanescentes ribeirinhos nas fazendas produtoras de Pinus em estudo evidenciou a função de "gatilhos ecológicos" que estes núcleos de diversidade possuem na paisagem. Estes remanescentes mostraram uma capacidade de regeneração correspondente a capacidade natural da própria paisagem. Por outro lado, o uso de técnicas nucleadoras mostrou a possibilidade de acelerar o processo sucessional, resgatando não só aspectos estruturais, mas também a funcionalidade entre os organismos da comunidade local. Os núcleos de solo com o banco de sementes de fragmentos conservados deverão ser internalizados na área, resgatando parte dos atributos e funções de um solo originalmente conservado e as interações entre organismos. Já os poleiros artificiais permitiram evidente aumento da biodiversidade local, indicando a importância de estabelecer pontos de ligação entre áreas abertas e fragmentos conservados.

A atividade de restauração, tendo como princípio básico a nucleação, tende a facilitar o processo sucessional natural, tornando-se mais efetiva quanto mais numerosos e diversificados forem os núcleos, pois as ações nucleadoras se complementarão no sentido de rapidamente formar uma comunidade mais estabilizada (Reis et al., 2007). Essas estratégias representam a oportunidade de restaurar a conectividade local e de contexto da paisagem onde os processos ecológicos sejam mantidos e capazes de garantir a resiliência ambiental.

\section{Agradecimentos}

A Empresa Battistella Florestal, Rio Negrinho, SC, pelo apoio logístico e financeiro para o desenvolvimento da pesquisa. À CAPES pela concessão de bolsa de mestrado à primeira autora. 


\section{Referências}

Bascompte, J.; Jordano, P.; Olesen, J. M. 2006. Asymetric coevolutionary networks facilitate biodiversity maintenance. Science, 312: 431-433.

Basso, S.; Langa, R.; Ribas Jr, U.; Tres, D. R.; Scariot, E. C.; Reis, A. 2007. Introdução de Mimosa scabrella em áreas ciliares através da transposição de amostras de solo. Revista Brasileira de Biociências, 5 (1): 684-686

Bechara, F. C. 2003. Restauração ecológica de restingas contaminadas por Pinus no Parque Florestal do Rio Vermelho, Florianópolis, SC. Dissertação de Mestrado, Universidade Federal de Santa Catarina, Brasil, 125pp.

Bechara, F. C. 2006. Unidades demonstrativas de restauração ecológica através de técnicas nucleadoras: Floresta Estacional Semidecidual, Cerrado e Restinga. Tese de Doutorado, ESALQUSP, Brasil, 248pp.

Bechara, F. C.; Campos Filho, E. M.; Barretto, K. D.; Gabriel, V. A.; Antunes, A. Z.; Reis, A. 2007a. Unidades demonstrativas de restauração ecológica através de técnicas nucleadoras de biodiversidade. Revista Brasileira de Biociências, 5: 9-11.

Bechara, F. C.; Fernandes, G. D.; Silveira, R. L. 2007b. Quebra de dormência de sementes de Chamaecrista flexuosa (L.) Greene Leguminosae visando à restauração ecológica do Cerrado. Revista de Biologia Neotropical, 4: 58-63.

Beiguelman, B. 1991. Curso Prático de Bioestatística. Sociedade Brasileira de Genética, Ribeirão Preto, Brasil, 224pp.

Benayas, J. M. R.; Bullock, J. M.; Newton, A. C. 2008. Creating woodland islets to reconcile ecological restoration, conservation, and agricultural land use. Frontiers in Ecology and the Environment, 6: 329-336.

Bradshaw, A. D. 1983. The reconstruction of ecosystems. Journal of Applied Ecology, 20: 1-17.

Castro, J.; Zamora, R.; Hódar, J. A.; Gómez J. M.; Gómez-Aparicio, L. 2004. Benefits of using shrubs as nurse plants for reforestation in Mediterranean mountains: a 4-year study. Restoration Ecology, 12: 352-358.

Christoffoleti, P. J.; Caetano, R. S. X. 1998. Soil seed banks. Scientia Agrícola, 55: 1-7.

Cubina, A.; Aide, T. M. 2001. The effect of distance from Forest edge on seed rain and soil seed bank in a tropical pasture. Biotropica, 32: 260-267.

Dobson, A. P.; Bradshaw, A. D.; Baker, A. J. M. 1997. Hopes for the future: restoration ecology and conservation biology. Science, 277: 515-522.

Espindola, M. B. 2005. O papel da chuva de sementes na restauração da restinga no Parque Florestal do Rio Vermelho, Florianópolis, SC. Dissertação de Mestrado, Universidade Federal de Santa Catarina, Brasil, 54pp.

Espindola, M. B.; Vieira, N. K.; Reis, A.; Hmeljevski, K. V. 2003 Poleiros artificiais: formas e funções. Disponível em $<\mathrm{http}$ :/www. sobrade.com.br/eventos/2003/seminário/Trabalhos/trabalhos. htm>. Acesso em 13 de dezembro de 2008.

Ferreira, P. V. 1991. Estatística experimental aplicada à agronomia. EDUFAL, Maceió, Brail, 440pp.

Fundação SOS Mata Atlântica/Instituto Nacional de Pesquisas
Espaciais/Instituto Socioambiental. 1998. Atlas da evolução dos remanescentes florestais e ecossistemas associados do domínio da Mata Atlântica no período 1990-1995. Fundação SOS Mata Atlântica, São Paulo, Brasil, 29pp.

Garcia, D.; Zamora, R. 2003. Persistence, multiple demographic strategies and conservation in long-lived Mediterranean plants. Journal of Vegetation Science, 14: 921-926.

Garcia, D.; Zamora, R.; Hódar, J. A.; Gómez, J. M; Castro, J. 2000. Yew (Taxus baccata L.) regeneration is facilitated by fleshy-fruited shrubs in Mediterranean environments. Biological Conservation, 95: $31-38$.

Gómez-Aparicio, L.; Zamora, R.; Gomez, J. M.; Hodar, J. A.; Castro, J.; Baraza, E. 2004. Applying plant facilitation to fores restoration: A meta-analysis of the use of shrubs as nurse plants. Ecological Applications, 14: 1128-1138.

Grant, V. 1980. Gene flow and the homogeneity of species populations. Biologisches Zentralblatt, 99: 157-169.

Griffith, J. J.; Toy, T. J. 2005. O modelo físico-social da recuperação ambiental. Brasil Mineral, 242: 166-174.

Guinle, M. C. T. 2006. Sucessão secundária da vegetação ciliar na micro-bacia do Rio Verde, Rio Negrinho, SC. Dissertação de Mestrado, Universidade Federal de Santa Catarina, Brasil, 78pp.

Hmeljevski, K. V. 2004. Levantamento florístico de restinga contaminada por Pinus spp. no Parque Florestal do Rio Vermelho, Florianópolis, SC. Trabalho de Conclusão de Curso, Universidade Federal de Santa Catarina, Brasil, 25pp.

Hurlbert, S. 1971. The nonconcept of species diversity: A critic and alternative parameters. Ecology, 52 (4): 577-586.

Janzen, D. H. 1970. Herbivores and the number of tree species in Tropical Forests. American Naturalist, 104: 501-528.

Klein, R. M. 1985. Os tipos florestais com Araucária em Santa Catarina. Anais do XXXVI Congresso Brasileiro de Botânica, Curitiba, Brasil, p.97-100.

Ludwig, J. A.; Reinolds, J. F. 1988. Statistical ecology: A primer on methods and computing. Wiley-Interscience, New York, USA, 337 pp.

Mcintosh, R. P. 1980. The relationship between succession and the recovery process in ecosystems. In: Cairns Jr., J. (Ed.). The recovery process in damaged ecosystems. Ann Arbor: Ann Arbor Science Publishers, Michigan, USA, p.11-62.

Metzger, J. P. 2006. How to deal with non-obvious rules for biodiversity conservation in fragmented areas. Natureza e Conservação, 4: 125-137.

Moilanen, A.; Hanski, I. 2001. On the use of connectivity measures in spatial ecology. Oikos, 95: 147-151.

Moreira, F. M. S.; Siqueira, J. O. 2002. Microbiologia e bioquímica do solo. UFLA, Lavras, Brasil, 626pp.

Palmer, M. A.; Ambrose, R. F.; Poff, N. L. 1997. Ecological theory and community restoration ecology. Restoration Ecology, 5: 291300 .

Parker, T. V.; Pickett, S. T. A. 1999. Restoration as an ecosystem process: Implications of the modern ecological paradigm. In: Urbanska, K.; Webb, N. \& Edward, P. (Eds). Restoration ecology and sustainble development. Cambridge University Press, Cambridge, UK, p.17-32. 
Pijl, L. V. 1982. Principles of dispersal in higher plants. SpringerVerlag, Berlim, Germany, 162pp.

Reis, A.; Bechara, F. C.; Espíndola, M. B.; Vieira, N. K.; Souza, L. L. 2003. Restoration of damaged land areas: Using nucleation to improve sucessional processes. The Brazilian Journal of Nature Conservation, 1 (1): 85-92.

Reis, A.; Tres, D. R. 2007. Nucleação: Integração das comunidades naturais com a paisagem. In: Fundação Cargill (Ed.). Manejo ambiental e restauração de áreas degradadas. Cargill, São Paulo, Brasil, p.28-55.

Reis, A.; Tres, D. R.; Scariot, E. C. 2007. Restauração na Floresta Ombrófila Mista através da sucessão natural. Pesquisa Florestal Brasileira, 55: 67-73.

Reis, A.; Zambonin, R. M.; Nakazono, E. M. 1999. Recuperação de áreas florestais degradadas utilizando a sucessão e as interações planta-animal. Série Cadernos da Biosfera, 14: 1-42.

Rosenzweig, M. L. 1995. Species diversity in space and time. Cambridge University Press, Cambridge, UK, 436pp.

Santa Catarina. 1986. Atlas de Santa Catarina. Aerofoto Cruzeiro, Rio de Janeiro, Brasil, 36pp.

SER - Society for Ecological Restoration International Science \& Policy Working Group. 2004. The SER International Primer on Ecological Restoration. $1^{\text {st }}$ ed. SER, Tucson, USA, 14pp.

SER - Society for Ecological Restoration International; IUCN - International Union for Conservation of Nature and Natural Resources. 2004. Ecological restoration, a means of conserving biodiversity and sustaining livelihoods. SER and IUCN, Tucson, USA, 6pp.

Sokal, R. R.; Rohlf, F. J. 1995. Biometry: The principles and practice of statistics in biological research. W. H. Freeman and Company, New York, USA, p.446-450.

Stewart, A. J. A.; John, E. A.; Hutchings, M. J. 2002. The world is heterogeneous: Ecological consequences of living in a patchy environment. In: Hutchings, M. J.; John, E. A. \& Stewart, A. J. A. (Eds). The ecological consequences of environmental heterogeneity. Cambridge University Press, Cambridge, UK, p.1-8.

Tres, D. R.; Guinle, M. C. T.; Reis, A.; Basso, S.; Langa, R.; Ribas Jr., U. 2005. Uso de técnicas nucleadoras para restauração ecológica de matas ciliares, Rio Negrinho, SC. Anais do Simpósio Nacional e Congresso Latino-Americano sobre Recuperação de Áreas Degradadas, Curitiba, Brasil, p.71-79.

Tres, D. R. 2006. Restauração ecológica de uma mata ciliar em uma fazenda produtora de Pinus taeda L. no norte do Estado de Santa Catarina. Dissertação de Mestrado, Universidade Federal de Santa Catarina, Brasil, 85pp.
Tres, D. R.; Reis, A. 2007. La nucleación como propuesta para la restauración de la conectividad del paisaje. Anais do II Seminário Internacional de Restauración Ecológica, Santa Clara, Cuba, p.32-42.

Tres, D. R., Sant'Anna, C. S., Basso, S., Langa, R., Ribas Jr., U., Reis, A. 2007a. Banco e Chuva de Sementes como Indicadores para a Restauração Ecológica de Matas Ciliares. Revista Brasileira de Biociências, 5: 309-311.

Tres, D. R., Sant'Anna, C. S., Basso, S., Langa, R., Ribas Jr., U., Reis, A. 2007b. Poleiros Artificiais e Transposição de Solo para a Restauração Nucleadora em Áreas Ciliares. Revista Brasileira de Biociências, 5: 312-314.

Vieira, N. K. 2004. O papel do banco de sementes na restauração de restinga sob talhão de Pinus elliottii Engelm. Dissertação de Mestrado, Universidade Federal de Santa Catarina, Brasil, 75pp.

Walker, L. R.; Del Moral, R. 2003. Primary succession and ecosystem rehabilitation. University Press, Cambridge, UK, 442pp.

Whisenant, S. G. 1999. Repairing damaged wildlands: A process-orientated, landscape-scale approach. University Press, Cambridge, UK, 224pp.

Williams, R.; Martinez, N. 2000. Simple rules yield complex food webs. Nature, 404: 180-183.

Wilson, S. D. 2002. Heterogeneity, diversity and scale in plant communities. In: Hutchings, M. J.; John, E. A. \& Stewart, A. J. A. (Eds). The ecological consequences of environmental heterogeneity. Cambridge University Press, Cambridge, UK, p.5269.

Yarranton, G. A.; Morrison, R. G. 1974. Spatial dynamics of a primary succession: nucleation. Journal of Ecology, 62 (2): 417428

Young, T. P. 2000. Restoration ecology and conservation biology. Biological Conservation, 92: 73-83.

Young, T. P.; Chase, J. M.; Huddleston, R. T. 2001. Community succession and assembly: comparing, contrasting and combining paradigms in the context of ecological restoration. Ecological Restoration, 19: 5-18.

Zamora, R.; García-Fayos, P.; Gómez-Aparicio, L. 2004. Las interacciones planta-planta y planta-animal en el contexto de la sucesión ecológica. In: Valladares, F. (Ed.). Ecología del bosque mediterráneo en un mundo cambiante. EGRAF, Madrid, España, p. 371-393. 